\title{
BMJ Open Inequalities in full vaccination coverage based on maternal education and wealth quintiles among children aged 12-23 months: further analysis of national cross-sectional surveys of six South Asian countries
}

\author{
Kiran Acharya (D) , ${ }^{1}$ Dinesh Dharel, ${ }^{2}$ Raj Kumar Subedi, ${ }^{3}$ Asmita Bhattarai, ${ }^{4}$ \\ Yuba Raj Paudel (i) ${ }^{5}$
}

To cite: Acharya K, Dharel D, Subedi RK, et al. Inequalities in full vaccination coverage based on maternal education and wealth quintiles among children aged 12-23 months: further analysis of national crosssectional surveys of six South Asian countries. BMJ Open 2022;12:e046971. doi:10.1136/ bmjopen-2020-046971

- Prepublication history and additional supplemental material for this paper are available online. To view these files, please visit the journal online (http://dx.doi.org/10.1136/ bmjopen-2020-046971)

Received 23 November 2020 Accepted 29 December 2021

Check for updates

(C) Author(s) (or their employer(s)) 2022. Re-use permitted under CC BY-NC. No commercial re-use. See rights and permissions. Published by BMJ.

For numbered affiliations see end of article.

Correspondence to

Kiran Acharya;

acharya.kiran1@gmail.com

\section{ABSTRACT}

Objective This study was conducted to compare full vaccination coverage and its inequalities (by maternal education and household wealth quintile).

Design This further analysis was based on the data from national-level cross-sectional Demographic and Health Survey (DHS) from six countries in South Asia.

Setting We used most recent DHS data from six South Asian countries: Nepal, India, Pakistan, Bangladesh, Afghanistan and the Maldives. The sample size of children aged 12-23 months ranged from 6697 in the Maldives to 628900 in India.

Primary and secondary outcome measures To measure absolute and relative inequalities of vaccination coverage, we used regression-based inequality measures, slope index of inequality (SII) and the relative index of inequality (RII), respectively, by maternal education and wealth quintile. Results Full vaccination coverage was the highest in Bangladesh (84\%) and the lowest in Afghanistan (46\%), with an average of $61.5 \%$ for six countries. Pakistan had the largest inequalities in coverage both by maternal education (SIl: -50.0, RIl: 0.4) and household wealth quintile (SII: -47.1, Rll: 0.5 ). Absolute inequalities were larger by maternal education compared with wealth quintile in four of the six countries. The relative index of inequality by maternal education was lower in Pakistan (0.5) and Afghanistan (0.5) compared with Nepal (0.7), India (0.7) and Bangladesh (0.7) compared with rest of the countries. By wealth quintiles, RII was lower in Pakistan (0.5) and Afghanistan (0.6) and higher in Nepal (0.9) and Maldives (0.9).

Conclusions The full vaccination coverage in 12-23 months old children was below $85 \%$ in all six countries. Inequalities by maternal education were more profound than household wealth-based inequalities in four of six countries studied, supporting the benefits of maternal education to improve child health outcome.

\section{INTRODUCTION}

Countries have agreed to reach all children with immunisation services in the global commitments adopted as the 2030 agenda

\section{Strengths and limitations of this study}

We analysed data from the recent nationally representative Demographic and Health Survey (DHS) of six South Asian countries.

- The study used regression-based inequality measures, the slope index of inequality and the relative index of inequality by maternal education and wealth quintile.

- The DHS data could not capture the differences in the health system, cultural factors and demand for immunisation services that might affect full vaccination coverage.

- Vaccination data partially comes from maternal recall of children's vaccine receipt that may introduce recall bias.

- The DHS is a cross-sectional survey; it was not possible to establish a temporal relationship between childhood vaccination and explanatory factors.

for sustainable development. ${ }^{1}$ Additionally, the Global Vaccine Action Plan (GVAP) of the WHO had set global targets for all countries to achieve $90 \%$ national coverage of the third dose of diphtheria, pertussis, and tetanus (DPT3) vaccine and its $80 \%$ subnational coverage by $2015 .^{2}$

Although significant progress has been achieved in terms of these targets, the WHO/ UNICEF Estimates of National Immunization Coverage suggests that progress lags in both coverage and equity that differ across countries and regions. ${ }^{3}$ As of 2012, it was estimated that as many as one in four children in South Asia are not fully immunised. ${ }^{4}$ In 2018, there were 20 million unvaccinated or undervaccinated children worldwide. ${ }^{5}$ South Asia reported 8 million of the world's 23 million 
underimmunised children in 2012. ${ }^{4}$ The WHO's 2017 assessment of GVAP found that in some countries, children from families in the highest wealth quintile have a nine times greater chance of receiving three doses of DPT than those from the poorest wealth quintile. ${ }^{6}$ Literature suggests that South Asia suffers from the high disparity in terms of wealth and status of women, which are barriers to equity in terms of coverage of health indicators. ${ }^{7}$ Thus, the need to study disparities between and within the countries regarding vaccination coverage is quite evident.

Moreover, South Asian countries have close economic $\operatorname{ties}^{8}$ and have cultural and ethnic commonalities. ${ }^{9} 10$ There is high population mobility across these countries for trade, labour, tourism and family visits. For example, there is an open border policy between Nepal and India that allows citizens of both countries to move from one country to another for livelihood and important life events such as marriage and childbirth without requiring travel permits. Thus, it is important to achieve coverage of herd immunity threshold to achieve community protection and to protect citizens of the neighbouring country from contracting vaccine-preventable diseases. More importantly, as the pursuit of universal health coverage accelerates, countries need to strive to reach the unreached children. However, there is no study in South Asia comparing full vaccination coverage across countries and assessing inequalities by key measures of inequalities (household wealth quintile and maternal educational status). ${ }^{11}$

Thus, we used data from recent Demographic and Health Survey (DHS) from six South Asian countries (Nepal, India, Pakistan, Bangladesh, Afghanistan and the Maldives). The aim was to identify the inequalities in full vaccination coverage by maternal education and household wealth quintile within and among six countries.

\section{MATERIALS AND METHODS \\ Data}

We used data from the most recent DHS in six South Asian countries. These surveys were: DHS Bangladesh 2014; National Family Health Survey India 2015-2016; DHS Maldives 2016-2017; DHS Nepal 2016, DHS Afghanistan 2015 and DHS Pakistan 2017-2018. These surveys are comparable nationally representative household surveys that collect, analyse and disseminate accurate and representative data on population, health, HIV and nutrition and have been conducted in more than 90 countries. Data are representative at the national level, urban/rural residence level and typically at least one subnational regional/ provincial level. Sri Lanka and Bhutan are not included in this study because Bhutan has not yet conducted any DHS, and Sri Lanka has its own DHS without support from the DHS program since 1987. Also, Sri Lanka did not measure the full vaccination coverage among 12-23 months of children in their recent DHS survey in 2016. The total sample size of the DHS surveys included in this study ranged from 6697 (Maldives) to 628900 (India)
Table 1 Analytic sample size of children aged 12-23 months

\begin{tabular}{llc}
\hline Country & Survey year & $\begin{array}{l}\text { Children aged 12-23 } \\
\text { months }\end{array}$ \\
\hline Pooled & $2014-2018$ & 58707 \\
Nepal & 2016 & 1034 \\
Bangladesh & 2014 & 1633 \\
India & $2015-2016$ & 47839 \\
Maldives & $2016-2017$ & 518 \\
Pakistan & $2017-2018$ & 1975 \\
\hline Afghanistan & 2015 & 5708 \\
\hline
\end{tabular}

households. DHS surveys of all the countries employed multistage, clustered sampling and similar data collection methods. Details of the surveys and sampling strategy can be found in final survey reports of all countries published elsewhere. ${ }^{12-17}$ The results for the Maldives for maternal education should be interpreted with caution because the number of children with no maternal education in the sample was less than 25 .

Response rates of eligible women range from $92 \%$ to $99 \%$. This study focuses its analyses on children aged 12-23 months, and the primary respondents were their mother or caregivers. We used the DHS child file to capture the children aged 12-23 months. The analytic sample size of children aged 12-23 months for the pooled data was 58 707 , with individual countries shown in table 1.

\section{Variables}

Vaccination coverage

According to $\mathrm{WHO}$, full vaccination coverage among children refers to "having received a Bacille Calmette Guerin (BCG) vaccine, three doses of combination vaccine including at least diphtheria, pertussis, and tetanus (DPT), three doses of oral polio vaccines, and a dose of measles-containing vaccine' ${ }^{18-20}$ The data on full vaccination coverage is collected from mothers of children aged 12-23 months, by reviewing vaccination cards. In the case of the unavailability of vaccination cards at the household at the time of the survey, the assessment of full vaccination status relied on mothers' reports.

We identified the important predictor variables for vaccination coverage of children aged 12-23 months in the pooled sample. These predictors included: (1) sex of child, (2) maternal education, (3) wealth quintile, (4) residence, (5) birth order of the child, (6) mother's age (15-19/20-34/35-49) and (7) source of vaccination (vaccination card seen/vaccination card not seen, ie, mother's report). The source of vaccination, birth order of child, maternal education and wealth quintile were significantly associated with full vaccination coverage (online supplemental table S1). To measure inequity, driven by socioeconomic determinants and based on the theoretical framework of the Commission on Social Determinants of Health, ${ }^{21}$ we used maternal education 
and wealth quintile for inequality of vaccination coverage in South Asia.

\section{Markers and measures of inequality}

DHS collects information about household assets, dwelling characteristics and ownership of consumer items. Based on these items, DHS calculates a wealth index to estimate socioeconomic position. ${ }^{22}$ The highest educational attainment of each participant was classified based on the number of years of schooling as 'no education (0 years)', 'primary ( $1-5$ years)', 'secondary (6-10 years)' or 'higher ( $>10$ years, university or vocational education after school)'. Each participant was given a relative socioeconomic rank and categorised as belonging to one of five country-specific quintiles, where the first quintile (Q1) is the poorest $20 \%$ and the fifth quintile (Q5) the wealthiest $20 \%$. We report both absolute and relative measures of inequality since using either of the measures may lead to different conclusions about the magnitude of inequality. ${ }^{23}$

\section{Statistical analyses}

The study used the slope index of inequality (SII) and the relative index of inequality (RII), which are regressionbased inequality measures that take into account the size of the population across maternal education and wealth quintile. ${ }^{24} \mathrm{SII}$ is a complex weighted measure of inequality. It represents a difference between the estimated value of the most advantaged group and most disadvantaged groups, while also considering other groups. If its value is ' 0 ', then there is no inequality. The higher the value of SII (in either direction +ve or -ve), the higher the inequality. To calculate SII, we first calculated a weighted ranking considering the proportional distribution of population in each category of wealth quintile/maternal education.

We then calculated SII by regressing the midpoint of cumulative population distribution using a generalised linear model with a logit link. Its value indicates a difference in predicted values of the most advantaged and disadvantaged group. Although the confidence intervals (CIs) across education and wealth categories overlap, inequality exists when regressing the midpoint of cumulative distribution and expressing the difference between two extreme groups in the form of SII. While SII is the difference between estimated values of two extreme categories, RII is the ratio of these two categories. RII has the value of 1 , when there is no inequality. The farther the value is from 1 , the larger the inequality.

We calculated estimates of overall coverage (in percentage) and associated 95\% CIs for full vaccination coverage by category of maternal education and wealth quintiles for each country. To estimate inequalities of full vaccination coverage, we calculated the SII and RII using predicted marginal rates of full vaccination coverage. To enable comparison of the full vaccination coverage across maternal education and wealth quintiles, for each country and socioeconomic variable, that is, maternal education and wealth quintiles, we first calculated ridit scores indicating the cumulative proportion of the population at each socioeconomic level, ranked from the lowest to the highest. Individuals with the same score were assigned the average rank. We used logistic regression analyses and their marginal predictions to calculate SII and RII. We regressed full immunisation coverage as the outcome variable on the ridit score of maternal education and wealth quintile (separately) as the exposure variables. We then estimated marginal predictions and SEs of the full vaccination coverage at the bottom and top rank of the variable based on the model coefficients. The SII was expressed as the expected difference in full vaccination coverage between the poorest wealth quintile versus the wealthiest income quintile, and RII was expressed as the ratio of the same two estimates. ${ }^{25}$ Thus, SII $>0$ and RII $>1$ indicate that full vaccination coverage decreases with increasing socioeconomic position. Whereas SII $<0$ and RII $<1$ indicates full vaccination coverage increases with increasing socioeconomic position. Complex sampling design was used for the analysis using the "svy" command in STATA V.15.

\section{Ethical review}

DHS survey protocols undergo ethical review in the USA with the DHS programme's institutional review board. These surveys have undergone a second human subjects review with respective research ethics boards, namely, the Bangladesh Medical Research Council, Maldives National Health Research Committee, the National Bioethics Committee, the Pakistan Health Research Council and the Nepal Health Research Council. DHS in India and Afghanistan did not undergo separate ethics approval. Prior to the release, all survey data are anonymised.

\section{Patient and public involvement}

Patient/public was not involved throughout this secondary study.

\section{RESULTS}

The full vaccination coverage was the highest in Bangladesh (84\%), followed by Nepal (78\%) and Maldives $(76 \%)$ and was the lowest in Afghanistan (46\%). Average full vaccination coverage across the six South Asian countries studied was $61.5 \%$ (figure 1 ). The full vaccination coverage by maternal education and wealth quintiles in Nepal, Bangladesh, India, Maldives, Pakistan and Afghanistan are presented separately (online supplemental table S2). We have also presented the SII and RII for maternal education and wealth quintile separately in detail (online supplemental tables S3 and S4), respectively). Furthermore, the crude difference and crude ratios in full vaccination coverage between those with no education and other education categories and between those in the lowest wealth quintile versus other wealth categories are presented (online supplemental tables S3 and S4, respectively). 


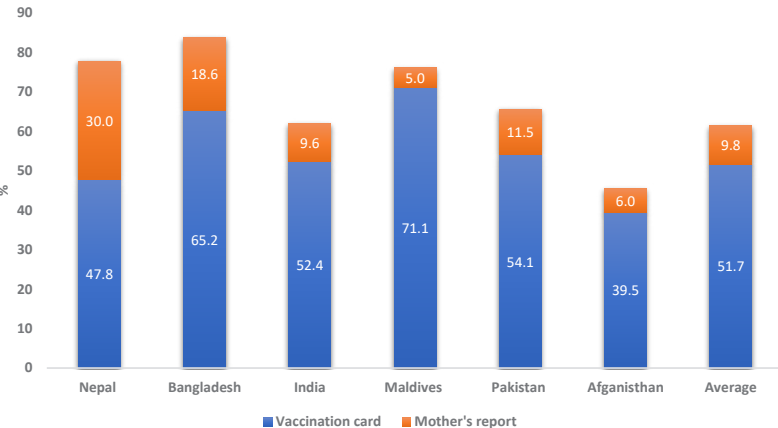

Figure 1 Full vaccination coverage in selected South Asian countries.

\section{Equity gap in full vaccination coverage among six South Asian countries}

The absolute difference in the predicted full vaccination coverage between children aged 12-23 months with no maternal education and higher maternal education was the largest in Pakistan $(\mathrm{SII}=-50.0)$, followed by Afghanistan (SII=-33.1), Nepal (SII=-30.5), Bangladesh (SII=-27.6) and India (SII=-24.3) (figure 2A). The results for Maldives for maternal education ( $\mathrm{SII}=-3.70)$ should be interpreted with caution because the number of children with no maternal education in the sample was less than 25. Likewise, the absolute difference in predicted full vaccination coverage between the wealthiest income quintile and the poorest quintile was the largest for Pakistan (SII=-47.1), followed by Bangladesh (SII=-27.6), Afghanistan (SII=-21.7) and India $(\mathrm{SII}=-20.7)$. At the same time, the difference was small for Nepal (SII=-8.4) and Maldives (SII=-8.4) (figure 3A). Furthermore, SII for wealth quintiles was lower than that for maternal educational status in all other countries except in Bangladesh and Maldives.

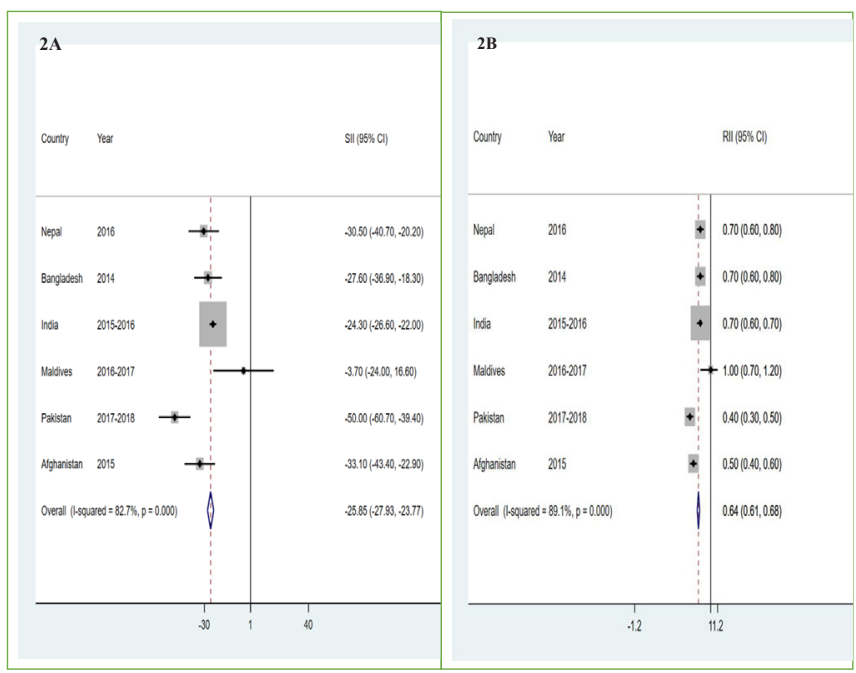

Figure 2 Maternal education-related inequalities. SII, slope index of inequality (2A); RII, relative index of inequality (2B).

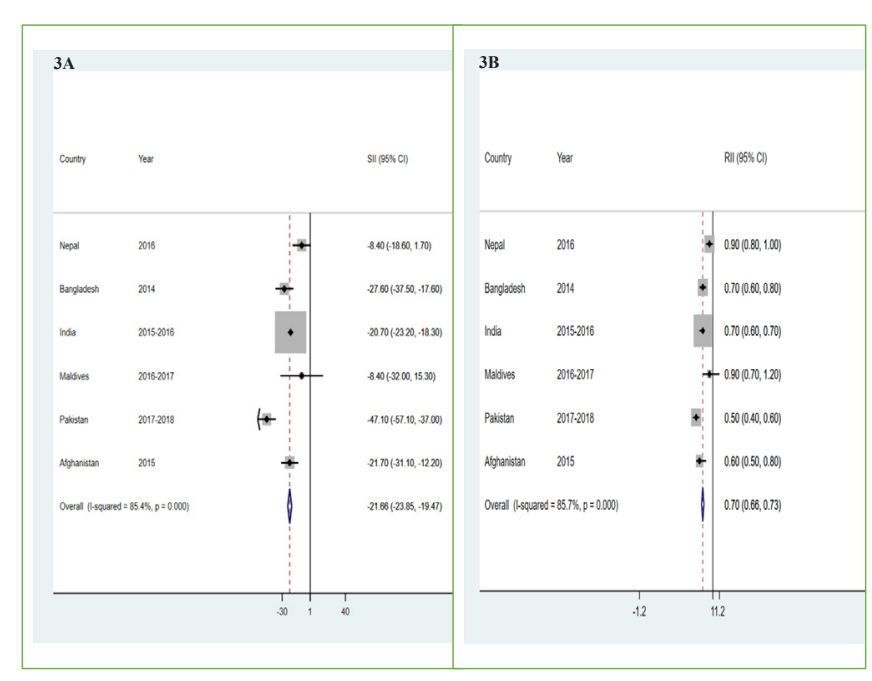

Figure 3 Wealth quintile-related inequalities. SII, slope index of inequality (3A); RII, relative index of inequality (3B).

The relative index of inequality of full vaccination coverage between children aged 12-23 months with no maternal education and higher maternal education was lower in Pakistan (RII=0.4, 95\% CI 0.3 to 0.5 ) and Afghanistan (RII $=0.5,95 \%$ CI 0.4 to 0.6) compared with Nepal (RII $=0.7,95 \%$ CI 0.6 to 0.8), Bangladesh (RII $=0.7$, $95 \%$ CI 0.6 to 0.8 ) and India (RII $=0.7,95 \%$ CI 0.6 to 0.7 ), indicating larger inequalities in Pakistan and Afghanistan driven by maternal educational status than in remaining countries (figure 2B). Similarly, the RII of full vaccination coverage between the lowest wealth quintile to the highest wealth quintile was low in Pakistan (RII $=0.5,95 \%$ CI 0.4 to 0.6 ) and Afghanistan (RII $=0.6,95 \%$ CI 0.5 to 0.8 ); intermediate in Bangladesh (RII $=0.7,95 \%$ CI 0.6 to 0.8 ) and India (RII $=0.7,95 \%$ CI 0.6 to 0.7$)$; and high in Nepal (RII $=0.9,95 \%$ CI 0.8 to 1.0 ) and Maldives (RII $=0.9$, $95 \%$ CI 0.7 to 1.2 ) (figure 3B). These findings suggest larger wealth-related inequalities in full immunisation coverage in Pakistan and Afghanistan than Maldives and Nepal, who had smaller wealth-related inequality in full vaccination coverage.

The analysis using absolute and relative indices of inequalities revealed some differences among the countries in the inequalities by maternal education and wealth quintiles (online supplemental tables S3 and S4, respectively). The relative inequalities in full vaccination coverage by wealth quintiles were lower in Nepal and the Maldives than in Afghanistan, Pakistan, Bangladesh and India (figure 3B). However, relative inequalities by maternal educational status were higher in Pakistan and Afghanistan compared with Nepal, India, Bangladesh and the Maldives (figure 2B). Pakistan had the highest absolute and relative inequalities both by maternal educational status (SII =-50.0) and household wealth quintiles $(\mathrm{SII}=-47.1)$. India has near average inequality in the region for both maternal education and household wealth quintile (figures 2 and 3), as expressed by both 


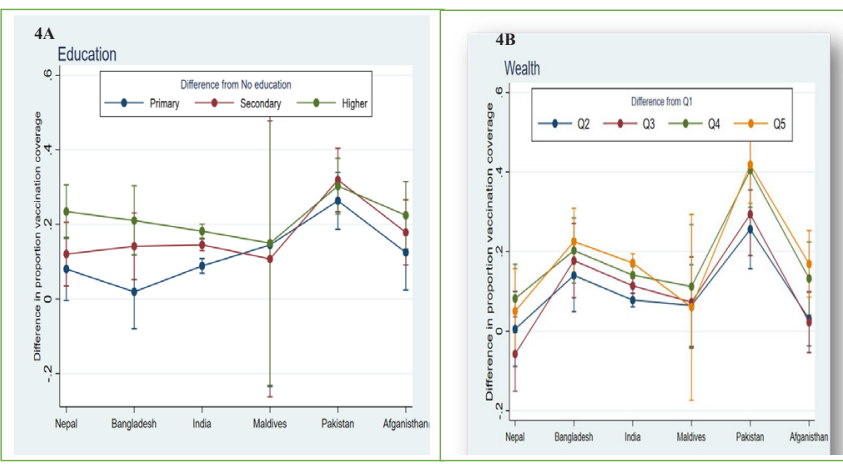

Figure 4 Crude prevalence difference in full vaccination coverage in South Asia by maternal education (4A) and wealth quintile (4B).

SII and RII. It is interesting to note that wealth-related inequalities in Afghanistan were slightly higher than in India and lower than in Bangladesh (online supplemental tables S3 and S4).

Similarly, the crude difference in full vaccination coverage between those with no maternal education and those with higher maternal education was the largest in Pakistan (30\%) and the smallest in India (18\%), excluding the Maldives (figure 4A). The crude difference in full vaccination coverage for the lowest wealth quintile was the largest in Pakistan (42\%) and smallest in the Maldives (6\%) and Nepal (5\%) (figure 4B). The detailed findings on the crude differences are shown in online supplemental table S3.

\section{DISCUSSION}

Current analysis of nationally representative DHS survey data from six South Asian countries demonstrated inadequate full vaccination coverage in the region ranging from $45.5 \%$ in Afghanistan to $83.8 \%$ in Bangladesh. Similarly, inequalities in vaccination coverage by mother's education and wealth quintile varied across countries. For example, the difference in maternal educational status creates much larger inequalities in vaccine coverage than household wealth differences in Nepal, Afghanistan and Pakistan. Similarly, in Pakistan, Bangladesh and Afghanistan, high inequalities in vaccination coverage are found by wealth quintiles compared with other South Asian countries. Overall, we have demonstrated that mothers' education is a stronger driver of inequitable full vaccination coverage than household wealth quintile in six countries from South Asia. The absolute difference in the predicted full vaccination coverage when mothers had no education compared with higher education ranged from SII of -24.3 in India to -50.0 in Pakistan. Although the SIIs of full vaccination among families from the lowest wealth quintile to those of the highest quintile were $-47.1,-27.6$, -21.7 and -20.7 in Pakistan, Bangladesh Afghanistan and India respectively, income was not a strong driver of inequitable vaccination coverage in Nepal and Maldives. The same applies to other countries, although at a smaller size. These findings are consistent with the literature showing the differential effect of maternal education and income on the uptake of preventive healthcare services. ${ }^{26}$ It has long been established that mothers' education is a strong determinant of child health-seeking behaviour. Better education is linked with improved decision-making status, lowered fertility, improved nutritional status and increased uptake of preventive health services such as vaccination. ${ }^{27}$

The average coverage of $61.5 \%$ across the six countries was almost 25\% lower than the global average of DPT3 coverage $(86 \%)$ in $2018 .{ }^{28}$ India and Pakistan, the two most populated countries in the region, had $62.0 \%$ and $61.5 \%$ coverage, respectively. This inadequacy contributes to a considerable portion of infants who are unimmunised or underimmunised in the region. Among 19.5 million children who did not receive the third dose of DPT, 11.8 million (nearly 60\%) lived in 10 countries that included India and Pakistan from the South Asia region. ${ }^{29}$ Nepal's full vaccination coverage increased remarkably from $65.6 \%$ in 2001 to $87.0 \%$ in 2011 and sharply decreased to $77.8 \%$ in 2016 , mainly due to a drop in DPT3 coverage. ${ }^{30}$ Although full vaccination coverage has recently decreased, ${ }^{30}$ Nepal's immunisation programme successfully narrowed inequities, mainly in terms of household wealth quintile and maternal education. ${ }^{30}{ }^{31}$ Bangladesh had the highest full vaccination coverage among the six countries. Bangladesh has been leveraging a pluralistic health system, emphasising women-centred, gender-equity oriented and focused maternal and child health programmes. ${ }^{9}$ Boulton $e t a l^{32}$ analysed the 2014 Bangladesh DHS data and reported that children from the poorest wealth quintile and born to women with low autonomy were significantly less likely to receive full vaccination, whereas maternal education was not associated. The current study has similar findings. We found that Bangladesh had higher absolute inequalities by wealth quintile than the other four countries (Nepal, India, Maldives and Afghanistan), while it had lower inequalities by maternal education compared to Nepal, Pakistan and Afghanistan.

Nepal has the second highest full vaccination coverage in the region and has the lowest inequality by household wealth quintile in terms of SII and RII. India, however, despite lower full vaccination coverage than most of the study countries (except Afghanistan), has near average inequality in the region for both maternal educational status and household wealth quintile. Nepal and India were chosen as two of the three (Senegal being the third) exemplary countries in the vaccine exemplar project funded by The Bill \& Melinda Gates Foundation that were hailed as the most successful countries for significantly improving vaccination coverage. ${ }^{33}$ Our analysis revealed that Maldives, India and Nepal have the most successful immunisation programme in the region in terms of equitable vaccination coverage by household 
wealth quintile and maternal education. However, all countries in the region, including Nepal and India, need concerted efforts to increase overall vaccination coverage further and reduce persisting inequities.

In our study countries, the routine immunisation services are provided free of cost to under-five children with funding support from the Vaccine Alliance. ${ }^{34}$ However, the role of wealth quintile on vaccination inequalities was seen in some countries. For example, In the case of Pakistan, Bangladesh and Afghanistan, high inequalities in vaccination coverage by wealth quintile may have been confounded by the variation in the decision-making status of the women among those from the different income groups. ${ }^{32} 35$ Besides, Pakistan and Afghanistan had a relatively lower ratio of full vaccination coverage among mothers with no education to those with high education compared with Nepal, Bhutan and Bangladesh. This resonates with the findings by Johri et $a l,{ }^{37}$ who demonstrated a high level of health literacy and improved communication skills among mothers with higher education, leading to increased odds of childhood immunisation. Moreover, researchers have also shown that people are geographically segregated by income with high-income households living in urban areas and low-income households living in rural areas. ${ }^{38}$ Thus, the relationship between income and childhood vaccination could have been confounded by geography, where access might be the underlying issue.

There are different barriers to achieving full immunisation in South Asian counties. Some of the major barriers are a weak health system, low demand for vaccination among the children's caregivers and small fiscal allocation in vaccination services. ${ }^{4}$ The national immunisation programmes in Nepal, Bhutan and Bangladesh have focused on the 'Reaching Every Community (REC)' approach, ${ }^{4}$ emphasising equitable access to vaccination services. ${ }^{39}$ This approach might be linked to better microplanning of immunisation services that increased coverage with narrower equity gap, particularly by income level in these countries compared with Pakistan and Afghanistan. A case study from India showed that an intensified campaign involving communities, multiple sectors, decentralised planning and implementation, and a high level of political commitment contributed to a significant increase in full immunisation coverage $(50.5 \%-69.0 \%)$ in low-performing districts. ${ }^{40}$

Afghanistan had much lower full vaccination coverage $(45.5 \%)$ than its neighbouring country Pakistan (65.6\%). However, inequities by maternal education and household wealth quintiles were higher in Pakistan than in Afghanistan. A community-based cross-sectional survey showed that nearly one-third $(31 \%)$ of the children were partially vaccinated in Afghanistan. ${ }^{41}$ Pakistan and Afghanistan are the only countries in the world where the wild poliovirus is endemic. ${ }^{42}$ Immunisation programmes in these countries face obstacles from lack of awareness on the need for vaccination, lack of faith and misconception about immunisation. ${ }^{41}$ Resistance from Islamic militants is much higher in Pakistan than in Afghanistan, leaving specific pockets in remote areas out of reach of immunisation network. ${ }^{43}$ Given religious opposition in these countries, information, education and behavioural communication strategies need to be contextualised by garnering the trust of religious leaders and community people. ${ }^{43}$ Misinformation regarding vaccines (causes infertility, is non-halal) needs to be addressed, and the polio eradication programme should be integrated into the routine immunisation programmes. ${ }^{44}$ Apart from inequalities results, our study from the pooled country data using logistic regression shows the high association between vaccination card seen and vaccination coverage. This finding here is similar to results found in other studies. $^{45-47}$

These study findings have implications for policies and programmes to increase full vaccination coverage and narrow the equity gap to meet the goals of universal coverage in the South Asia region. Policymakers need to prioritise sectoral policies to improve economic status or maternal education based on the relative importance of these sectors in their countries to improve full vaccination coverage. National health system programmers from study countries need to target the most disadvantaged children for full vaccination coverage. Implementing targeted programmes in the worst performing areas by strengthening local capacities may produce sustainable results. ${ }^{40}$

Making a cross-country comparison in terms of socioeconomic determinants of health is challenging when there are differences in data collection approaches, explanatory variables and statistical calculations across countries. However, such comparisons are valuable in informing regional and global policies in universal public health interventions such as immunisation programmes. Similarly, measuring health inequalities across socioeconomic groups involves judgements regarding population size, reference points for measuring departures from equality and the scale of inequality measurement. The strength of this study includes using most recent nationally representative demographic health surveys from the six countries of South Asia. The inequality is assessed based on the two most important determinants of preventive child health services: maternal education and wealth quintile using regression-based inequality measures: the SII and RII. The regression-based measures (SII and RII) are able to account for the intermediate ranking groups as well as the distribution of the population among different groups. However, they provide less 'information about the distribution of health status according to the distribution of the population or to the socioeconomic status of populations'. ${ }^{8}$ Although we used DHS data that have standardised methods and tools for data collection, the degree of comparability in our findings on inequality measures across countries might be low due to the large geography we are covering in this study. ${ }^{49}$

Some other limitations of this study are worth mentioning. The differences in the health system, 
cultural factors and demand for immunisation services might have affected full vaccination coverage in these countries, which we could not capture using the DHS data. Besides, other country-specific factors such as leadership, economy, topography, etc could have played crucial roles in creating differences in the vaccination coverages. Vaccination data are limited in that it partially comes from information contained on vaccination cards and partially from maternal recall may include recall bias. Furthermore, the DHS is a cross-sectional survey; it was not possible to establish a temporal relationship between childhood vaccination and explanatory factors. Despite these limitations, the DHS data allow for comparisons over the countries, especially since the definition of the outcome variable, that is, if the child received a specific vaccine or not, is easy to define, measure and compare.

\section{CONCLUSION}

The success of the global quest for universal childhood vaccination depends largely on the ability of low-income and middle-income countries to achieve high and equitable coverage. Our results inform the need to address such inequalities in South Asian countries by improving mother's educational status and household wealth quintile. These two determinants of vaccination inequality are also equally important in attaining the sustainable development goals.

\author{
Author affiliations \\ ${ }^{1}$ New ERA, Rudramati Marga, Kalopul, Kathmandu, Nepal \\ ${ }^{2}$ College of Medicine, University of Saskatchewan, Saskatoon, Saskatchewan, \\ Canada \\ ${ }^{3}$ Bhaskar Tejshree Memorial Foundation, Kathmandu, Nepal \\ ${ }^{4}$ Cumming School of Medicine, University of Calgary, Calgary, Alberta, Canada \\ ${ }^{5}$ School of Public Health, University of Alberta, Edmonton, Alberta, Canada
}

Acknowledgements The authors extend their heartfelt appreciation to USAID's Demographic and Health Survey programme for providing the datasets of six South Asian countries.

Contributors KA, DD and YRP were involved in the design and conception of the study. KA was involved in the analysis. YRP and KA interpreted the findings; $D D, R K S, A B, K A$ and YRP were involved in the write-up of the manuscript. All the authors supervised, reviewed and edited the manuscript. KA have access to the data and are responsible for the overall content as the guarantor. All authors read and approved the final manuscript.

Funding The authors have not declared a specific grant for this research from any funding agency in the public, commercial or not-for-profit sectors.

Competing interests None declared.

Patient consent for publication Not applicable.

Provenance and peer review Not commissioned; externally peer reviewed.

Data availability statement Data are available upon reasonable request. Data are available in a public, open access repository. The datasets generated during the current study are available from within the Demographic and Health Survey Program repository (https://dhsprogram.com/data/ available-datasets.cfm).

Supplemental material This content has been supplied by the author(s). It has not been vetted by BMJ Publishing Group Limited (BMJ) and may not have been peer-reviewed. Any opinions or recommendations discussed are solely those of the author(s) and are not endorsed by BMJ. BMJ disclaims all liability and responsibility arising from any reliance placed on the content. Where the content includes any translated material, BMJ does not warrant the accuracy and reliability of the translations (including but not limited to local regulations, clinical guidelines, terminology, drug names and drug dosages), and is not responsible for any error and/or omissions arising from translation and adaptation or otherwise.

Open access This is an open access article distributed in accordance with the Creative Commons Attribution Non Commercial (CC BY-NC 4.0) license, which permits others to distribute, remix, adapt, build upon this work non-commercially, and license their derivative works on different terms, provided the original work is properly cited, appropriate credit is given, any changes made indicated, and the use is non-commercial. See: http://creativecommons.org/licenses/by-nc/4.0/.

\section{ORCID iDs}

Kiran Acharya http://orcid.org/0000-0002-7757-0066

Yuba Raj Paudel http://orcid.org/0000-0003-3403-4359

\section{REFERENCES}

1 United Nations. Transforming our world: The 2030 agenda for sustainable development. General Assembley 70 session, 2015.

2 WHO. Global vaccine action plan 2011-2020, 2013.

3 World Health Organization. WHO-Vaccine preventable diseases: monitoring system. 2017, 2018.

4 Hasman A, Noble DJ. Childhood immunisation in South Asia - overcoming the hurdles to progress. Perspect Public Health 2016;136:273-7.

5 The United Nations Children's Fund. 20 million children missed out on lifesaving measles, diphtheria and tetanus vaccines in 2018, 2018 Available: https://www.unicef.org/press-releases/20-million-childrenmissed-out-lifesaving-measles-diphtheria-and-tetanus-vaccines [Accessed 06 Mar 2020].

6 World Health Organization. 2018 assessment report of the global vaccine action plan: strategic Advisory group of experts on immunization. World Health Organization, 2018.

7 Cunningham K, Ruel M, Ferguson E, et al. Women's empowerment and child nutritional status in South Asia: a synthesis of the literature. Matern Child Nutr 2015;11:1-19.

8 Saxena SC. Can South Asia adopt a common currency? J Asian Econ 2005; 16:635-62.

9 Chowdhury AMR, Bhuiya A, Chowdhury ME, et al. The Bangladesh paradox: exceptional health achievement despite economic poverty. Lancet 2013;382:1734-45.

10 Thapliyal S. Movement of population between India and Nepal: emerging challenges. Strategic Analysis 1999;23:777-89.

11 Arsenault C, Harper S, Nandi A, et al. An equity dashboard to monitor vaccination coverage. Bull World Health Organ 2017;95:128-34.

12 National Institute of Population Research, Training - NIPORT/ Bangladesh, Mitra. Bangladesh demographic and health survey 2014. Dhaka, Bangladesh: NIPORT, Mitra and Associates, and ICF International, 2016.

13 International Institute for Population Sciences, ICF. National family health survey (NFHS-4), 2015-16. India: IIPS Mumbai, 2017.

$14 \mathrm{MoH}$, ERA. N, ICF. Nepal demographic and health survey, 2016. Kathmandhu, Nepal: Ministry of Health, Nepal, 2017.

15 Ministry of Health - MOH/Maldives, ICF. Maldives demographic and health survey 2016-17. Malé, Maldives: MOH and ICF, 2018.

16 National Institute of Population Studies - NIPS/Pakistan, ICF. Pakistan demographic and health survey 2017-18. Islamabad, Pakistan, and Rockville, Maryland, USA: NIPS and ICF, 2019.

17 Central Statistics Organization/Afghanistan, Ministry of Public Health/ Afghanistan and, ICF. Afghanistan demographic and health survey, 2015. Kabul, Afghanistan: Central Statistics Organizations, 2017.

18 Mik S, Mf S, Kurlikar PR. Status and determinants of child immunisation coverage in three South Asian countries, India, Bangladesh and Nepal: evidence from the demographic and health survey. Sri Lanka J Child Heal 2018;47:56-63.

19 Animaw W, Taye W, Merdekios B, et al. Expanded program of immunization coverage and associated factors among children age 12-23 months in Arba Minch town and Zuria district, southern Ethiopia, 2013. BMC Public Health 2014;14:464.

20 Restrepo-Méndez MC, Barros AJ, Wong KL, et al. Inequalities in full immunization coverage: trends in low- and middle-income countries. Bull World Health Organ 2016;94:794-805.

21 Commision on Social Determinants of Health (CSDH). Background 3: key concepts. Geneva: World Health Organization, 2020. https:// www.who.int/social determinants/en/

22 Rutstein SO, Johnson K, MEASURE OM. The DHS wealth index: ORC macro, measure DHS, 2004.

23 Kunst AE, Mackenbach JP. Measuring socioeconomic inequalities in health. World Health Organization, 1990. 
24 Hosseinpoor AR, Nambiar D, Schlotheuber A, et al. Health equity assessment toolkit (heat): software for exploring and comparing health inequalities in countries. BMC Med Res Methodol 2016;16:141.

25 Moreno-Betancur M, Latouche A, Menvielle G, et al. Relative index of inequality and slope index of inequality: a structured regression framework for estimation. Epidemiology 2015;26:518-27.

26 Wamani H, Tylleskär T, Astrøm AN, et al. Mothers' education but not fathers' education, household assets or land ownership is the best predictor of child health inequalities in rural Uganda. Int $J$ Equity Health 2004;3:9.

27 Hobcraft J. Women's education, child welfare and child survival: a review of the evidence. Health Transit Rev 1993;3:159-75.

28 World Health Organization. Immunization coverage, 2019. Available: https://www.who.int/news-room/fact-sheets/detail/immunizationcoverage [Accessed 17 Feb 2020].

29 Feldstein LR, Mariat S, Gacic-Dobo M, et al. Global routine vaccination coverage, 2016. MMWR Morb Mortal Wkly Rep 2017;66:1252-5.

30 Acharya K, Paudel YR, Dharel D. The trend of full vaccination coverage in infants and inequalities by wealth quintile and maternal education: analysis from four recent demographic and health surveys in Nepal. BMC Public Health 2019;19:1673.

$31 \mathrm{Kc} \mathrm{A}$, Nelin V, Raaijmakers $\mathrm{H}$, et al. Increased immunization coverage addresses the equity gap in Nepal. Bull World Health Organ 2017;95:261-9.

32 Boulton ML, Carlson BF, Power LE, et al. Socioeconomic factors associated with full childhood vaccination in Bangladesh, 2014. Int J Infect Dis 2018;69:35-40.

33 Health EP, VIPs SV, 2019. Available: http://publichealthmagazine. emory.edu/issues/2019/spring/briefs/vaccine-VIPs/index.html [Accessed 17 Feb 2020].

34 Gavi:The Vaccine Alliance. Disbursements and commitments, 2020. Available: https://www.gavi.org/programmes-impact/our-impact/ disbursements-and-commitments [Accessed 17 Feb 2020].

35 Imran H, Raja D, Grassly NC, et al. Routine immunization in Pakistan: comparison of multiple data sources and identification of factors associated with vaccination. Int Health 2018;10:84-91.

36 Schütte S. Living with patriarchy and poverty: women's agency and the spatialities of gender relations in Afghanistan. Gender, Place \& Culture 2014;21:1176-92.
37 Johri M, Subramanian SV, Sylvestre M-P, et al. Association between maternal health literacy and child vaccination in India: a crosssectional study. J Epidemiol Community Health 2015;69:849-57.

38 Massey DS, Rothwell J, Domina T. The changing bases of segregation in the United States. Ann Am Acad Pol Soc Sci 2009;626:74-90.

39 Chan Soeung S, Grundy J, Duncan R, et al. From reaching every district to reaching every community: analysis and response to the challenge of equity in immunization in Cambodia. Health Policy Plan 2013;28:526-35.

40 Gurnani V, Haldar P, Aggarwal MK, et al. Improving vaccination coverage in India: lessons from intensified mission Indradhanush, a cross-sectoral systems strengthening strategy. BMJ 2018;363:k4782.

41 Mugali RR, Mansoor F, Parwiz S, et al. Improving immunization in Afghanistan: results from a cross-sectional community-based survey to assess routine immunization coverage. BMC Public Health 2017;17:290.

42 Mustafa S, Ghazal S, Waheed S, et al. Perspective on Polio Eradication - What is the Issue? Natl. j. health sci. 2019;4:3-6.

43 Kennedy J. Why have the majority of recent polio cases occurred in countries affected by Islamist militancy? A historical comparative analysis of the political determinants of polio in Nigeria, Somalia, Pakistan, Afghanistan and Syria. Med Confl Surviv 2016;32:295-316.

44 Habib MA, Soofi SB, Ali N, et al. Knowledge and perceptions of polio and polio immunization in polio high-risk areas of Pakistan. J Public Health Policy 2017;38:16-36.

45 Acharya K, Lacoul M, Bietsch K. Factors affecting vaccination coverage and retention of vaccination cards in Nepal. DHS further analysis reports no 121. Rockville, Maryland, USA: ICF, 2019.

46 Odusanya OO, Alufohai EF, Meurice FP, et al. Determinants of vaccination coverage in rural Nigeria. BMC Public Health 2008;8:1-8.

47 Chhabra P, Nair P, Gupta A, et al. Immunization in urbanized villages of Delhi. Indian J Pediatr 2007;74:131-4.

48 Alleyne GAO, Castillo-Salgado C, Schneider MC, et al. Overview of social inequalities in health in the region of the Americas, using various methodological approaches. Rev Panam Salud Publica 2002;12:388-97.

49 Mackenbach JP, Stirbu I, Roskam A-JR, et al. Socioeconomic inequalities in health in 22 European countries. $N$ Engl J Med Overseas Ed 2008;358:2468-81. 